\title{
Arrhythmia Detection Technique using basic ECG Parameters
}

\author{
Mohammad Rakibul Islam, Rifad Hossain, Md. Ziaul Haque Bhuiyan, Tahmeed Ahmed \\ Margoob, Md. Taslim Reza, Kazi Khairul Islam \\ Department of Electrical and Electronic Engineering, \\ Islamic University of Technology, Boardbazar, \\ Gazipur-1704, Bangladesh.
}

\begin{abstract}
A condition in which the heart beats with an irregular or abnormal rhythm is known as Arrhythmia. This paper presents a procedure to extract information from Electrocardiogram (ECG) data \& determine types of Arrhythmias. The decisions were achieved by determining different intervals such as PR Interval, RR Interval, Heart Rate (HR) etc. and those intervals were compared with the ideal intervals. During the whole process MATLAB was used \& ECG signals were taken from PhysioBank ATM. In this process Savitzky-Golay filter was used to reduce the noise of the signal. Tachycardia, Bradycardia, Heart Block, Junctional Arrhythmia, Premature Articular Contraction were detected during this analysis. The results show simplified detection of arrhythmia with $90 \%$ accuracy.
\end{abstract}

\section{General Terms}

Biomedical Signal Processing

\section{Keywords}

Electrocardiogram, Arrhythmia, PR Interval, RR Interval, Heart Rate.

\section{INTRODUCTION}

Arrhythmia is the abnormal rhythm of heart. It is also known as dysrhythmia. It causes the heart to pump less effectively. There are a lot of changes in the shape of the heart wave because of arrhythmia. ECG is a common term in the diagnosis of cardiac diseases. It provides information about the electrical activity of the heart. We can detect different kinds of heart diseases by analyzing the ECG signal. Higher efficiency in classifying ECG signal is very important nowadays. Detection of actual type of heart diseases is very important for further treatment. Heart disease is very dangerous for a human being. So, proper treatment is a must in this case. There are different sorts of arrhythmias. Different kinds of arrhythmias can be detected in different parts of the heart. Heart pumps blood in a regular way. But when it is affected by arrhythmia, it can't pump blood normally. Right bundle branch block (RBBB), left bundle branch block (LBBB), premature ventricular contractions (PVC), ventricular fibrillation (VF) are some serious arrhythmias. However ECG being a non-stationary signal, the irregularities may not be periodic and may not show up all the time, but would manifest at certain irregular intervals during the day. So, continuous ECG monitoring permits observation of cardiac variations over an extended period of time, either at the bad side or when patients are ambulatory, providing more information to physician. The heart rate and the morphology reflect the cardiac health of human heart beat [1]. It is a noninvasive method which means this signal is measured on the surface of human body, which is used in identification of the heart diseases [2]. Electrocardiography is the recording of the electrical activity of the heart. A typical ECG tracing of the cardiac cycle (heartbeat) consists of a $\mathrm{P}$ wave, a QRS complex, a $\mathrm{T}$ wave, and a $\mathrm{U}$ wave is shown in Fig. 1.

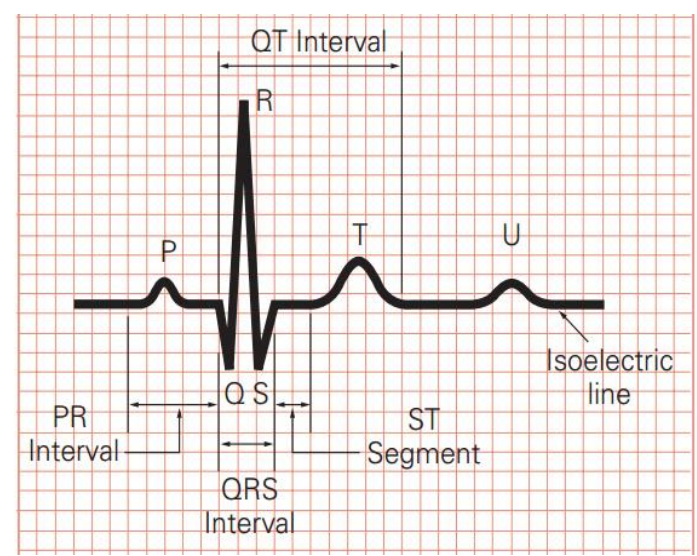

Fig. 1 Typical ECG Signal

For Arrhythmia detection we went through the Time Domain based technique. The technique is followed by ECG signal processing, determination of PR Interval, QRS Interval, QT Interval, ST segment, RR Interval (To determine Heart Rate) followed by Arrhythmia detection via some decision making rules. Table 1 shows normal ECG signal characteristics for different parameters. This paper is organized as follows. Section II describes different arrhythmia detection techniques where as in section III discuss problem formulation. ECG based arrhythmia detection technique is proposed in section IV. Results are discussed in section V whereas Section VI concludes the paper.

Table 1: Normal ECG Signal Characteristics

\begin{tabular}{|l|l|}
\hline Component & Characteristics \\
\hline Heart Rate & $60-100 \mathrm{bpm}$ \\
\hline PR Interval & $0.12-0.20 \mathrm{sec}$ \\
\hline QRS Interval & $0.06-0.10 \mathrm{sec}$ \\
\hline QT Interval & Less than half of the R-R interval \\
\hline ST segment & $0.08 \mathrm{sec}$ \\
\hline
\end{tabular}




\section{DIFFERENT ARRHYTHMIA DETECTION TECHNIQUES \\ 2.1 Fuzzy logic based techniques}

Fuzzy logic is a multivalued logic. It deals with intermediate values. This method is very efficient and it is similar to human thinking. Zadeh introduced this logic which can be used in many cases. It can be used for classifying ECG arrhythmias which is focused in this paper. This method can be used in modulation classifier in non-ideal environment which is very difficult or impossible by precise probabilistic methods [1]. Fuzzy if-then rules are used in many image processing applications [2]. This logic can be used in detecting H1N1 influenza. A hybrid method using Artificial Neural Network, Fuzzy Rule-base Classifier and Gaussian Mixture Model can be developed to detect H1N1 correctly [3].

Fuzzy rule-based classification system can be used in case of pattern classification problem. For this, error correction-based learning procedure and additional learning procedure is needed [4]. Type-2 Fuzzy sets also can be used for pattern recognition [5]. Genetic Fuzzy and Neuro-fuzzy classifier can be used for credit scoring. The Genetic Fuzzy classifier is more accurate in this case [6]. Again, Fuzzy classifier and fuzzy classified data are used in clinical proteomics. It is a very important tool for study at the protein and peptide level in medicine and health care [7]. Fuzzy rules are also applied in high-resolution multispectral satellite imagery for classification of urban and suburban areas [8].

\subsection{Fractal dimension based techniques}

Fractals are of rough or fragmented geometric shapes. These shapes can be sub divided in parts. Our conventional geometry deals with lines, triangles, circles, sphere, cones but the fractal geometry is concerned with fractured shapes. Fractals are often mentioned by their fractal dimension [9]. If we consider a fracture object whose mass is function of its radius but the radius doesn't go like $r^{2}$ and neither does like $r$ but rather it goes like a real power of $r$ between 1 and 2 . Such object with non-integer dimension is called fractals and the dimension of this type of object is called fractal dimension [10]. Fractal dimension measures the degree of fractal boundary fragmentation. It determines how the fractals differ from Euclidean objects. According to Mandelbrot, the relation between the data measuring scale $\delta$ and the length $\mathrm{L}$ can be expressed as [10]:

$$
\mathrm{L}(\delta)=\mathrm{k} \cdot \delta-\mathrm{D}
$$

Where, $\mathrm{K}$ is a constant and $\mathrm{D}$ is known is the fractal dimension. Fractal Dimension D is very often a non-integer number [11].

\subsection{Wavelet based packet techniques}

Discrete wavelet Transform (DWT) is multi-resolution transform with very fast implementation. DWT is a lost less linear transformation of a signal or data into coefficients on the basis of mother wavelet functions. A family of mother wavelet is available having the energy spectrum concentrated around the low frequencies. ECG signal is that kind of signal which is as well as better resembling the QRS complex of the ECG signal. Therefore, for the analysis of an ECG signal at different scales [12], wavelet transform (DWT) is frequently used in practice. In DWT, for analyzing both the low and high frequency components, it is passed through a series of low pass and high pass filters with different cut off frequencies. This process results in a set of approximate and detail DWT coefficients, respectively. The filtering operations in DWT result in a change in the signal resolution and thus, DWT decomposes the signal into approximate and detail information thereby helping in analyzing it. In wavelet analysis, only scale space is decomposed, but wavelet space is not. This results in a logarithmic frequency resolution [13], which does not work well for all the signals. By the restriction of Heisenberg's uncertainty principle, the spatial resolution and spectral resolution of high frequency band become poor and it limits the application of wavelet transform.

\subsection{Neural Network based techniques}

The electrocardiogram examination is widely used for diagnosis of cardiovascular diseases in clinical area for its non-invasiveness and reliability. In Intensive Care Unit (ICU), or Holter System, long-time recording and analyzing of ECG are required and automatic ECG pattern recognition techniques are used to reduce physicians' work and improve diagnosis efficiency. A typical ECG waveform contains P wave, QRS complex and T wave in each heart beat which is shown in Fig.1. Recognizing an ECG pattern is essentially the process of extracting and classifying ECG feature parameters, which can be obtained from either the time domain or transform domain. The features which are frequently used for ECG analysis in time domain include the wave shape, amplitude, duration, areas, and R-R intervals [14]. The basic problem of automatic ECG analysis occurs from the nonlinearity in ECG signals and the large variation in ECG morphologies of different patients. In most cases, ECG signals are contaminated by background noises, such as electrode motion artifact and electromyogram induced noise. It also add to the difficulty of automatic ECG pattern recognition [15].

\section{PROBLEM FORMULATION}

The Arrhythmia detection techniques mentioned above are complex in nature. Reduction in complexity remains the challenge now a day. The ECG of a normal heartbeat is compared with the heartbeats of different arrhythmias in Fig. 2.
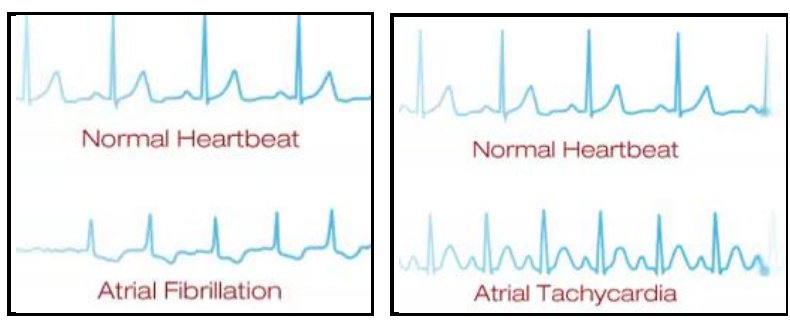

a) Atrial Fibriliation

b) Atrial Trachycardia

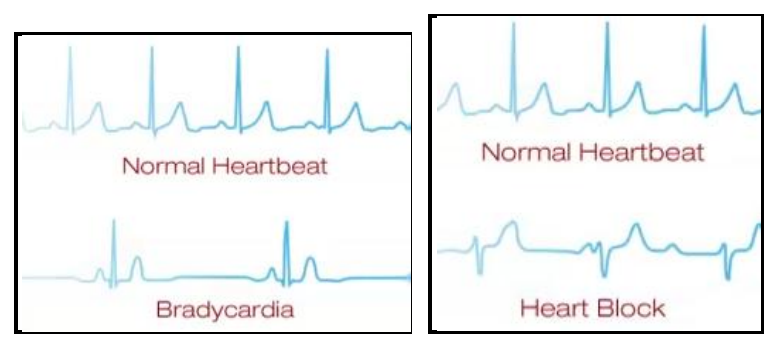

c) Bradycardia

d) Heart blck 


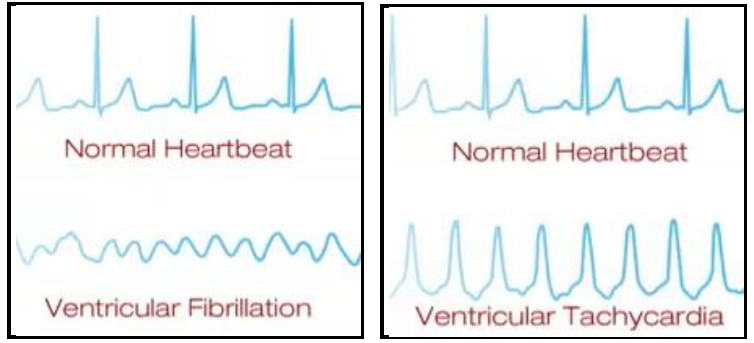

e) Ventricular Fibriliation f) Ventricular Trachycardia

Fig. 2: Normal Heartbeat vs. different arrhythmias

Previous diagrams show ECG characteristics of different sorts of arrhythmias which helps to understand the inner sight of arrhythmia.

This result motivates us to find the arrhythmia from basic ECG parameters [16].

\section{PROPOSED ECG BASED ARRHY- THMIA DETECTION}

The signals used in this process were taken from MIT-BIH database. The signals are sampled at $360 \mathrm{~Hz}$. From Figure 1, we can see a typical ECG signal showing P, Q, R, S, T \& U points including PR Interval, QRS Interval, QT Interval, ST Segment, Isoelectric line. The ECG parameters shown in Table 1 are important in finding the arrhythmia. Our proposed method is described using the flow diagram shown in Fig. 3.

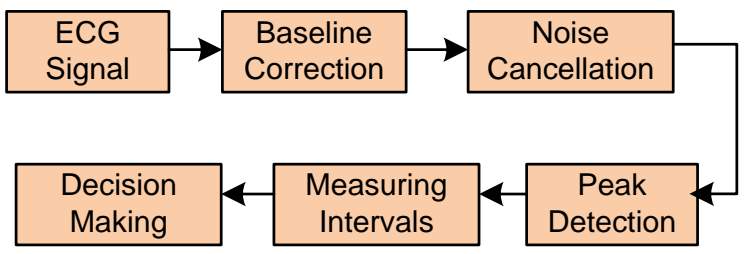

Fig. 3. Flow diagram for our proposed method

We start with the ECG signal shown in Figure 4. This is a noisy signal with variable baseline. Also the peaks cannot be detected easily. Using the following techniques, the ECG signal will be easily detectable.

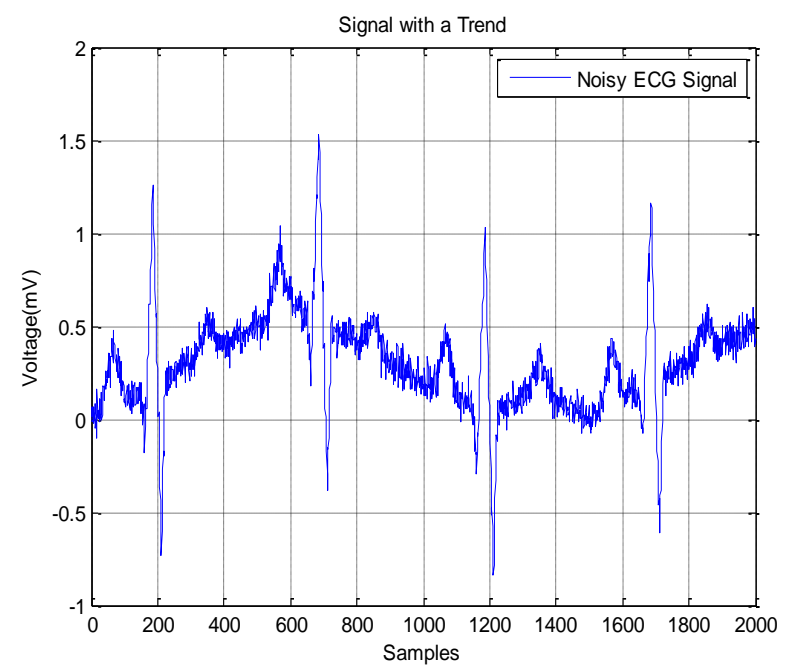

Fig. 4. Actual ECG Signal

\subsection{Baseline Correction and Signal Smoothing}

The original ECG Signal is fed through smoothing filters. At the output of the smoothing filter, baseline of the signals was corrected and is shown in Fig. 5.

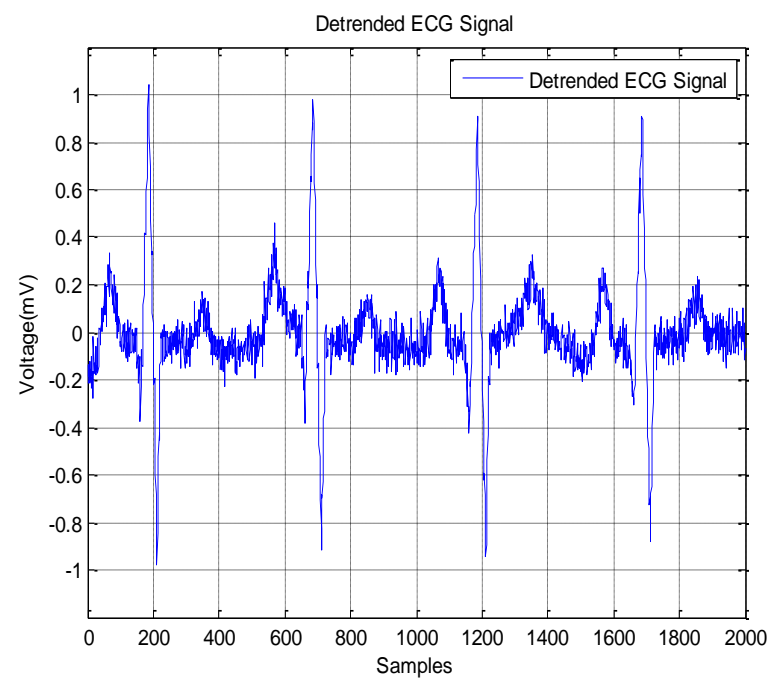

Fig. 5. Baseline Corrected ECG Signal

\subsection{Noise cancellation using Savitzky-Golay Filter}

Savitzkey-Golay filter is used to cancel the noise in the ECG signal. Savitzkey-Golay filter is widely known for its signal noise cancellation. The ECG signal after using SavitzkeyGolay filter is shown in Fig. 6.

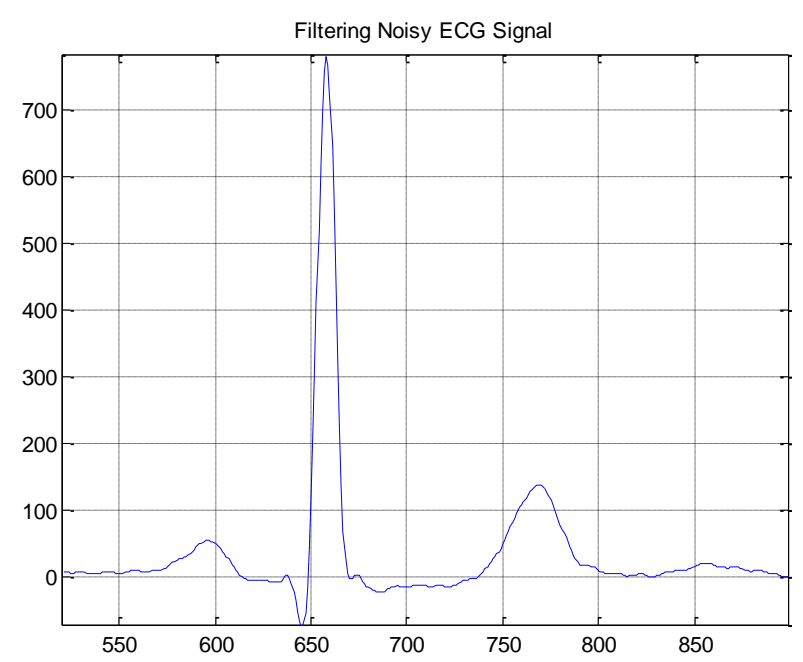

Fig. 6. Noise cancellation using Savitzky-Golay Filter

\subsection{Locating Peaks}

$\mathrm{P}, \mathrm{Q}, \mathrm{R}, \mathrm{S}, \mathrm{T}$ peak detection is a tough task. Especially, when the ECG signal is not normal, more than two peaks are found. We used a special algorithm to eliminate the unnecessary peaks. After using the algorithm, the program was able find the peaks in all conditions. Fig. 7 shows the detection of peaks. 


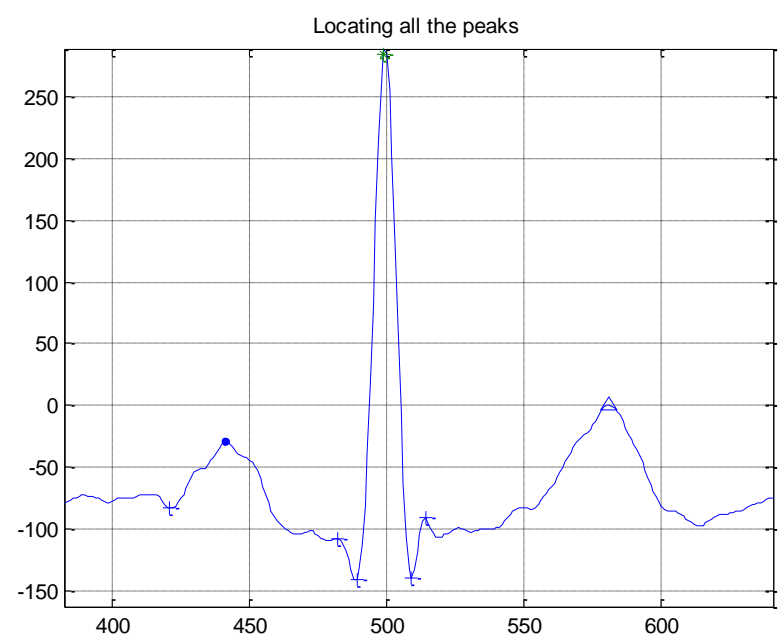

Fig. 7. Peak Detection

\subsection{Measuring Intervals}

After locating peak points, different intervals, such as: PR Interval, QRS Interval, QT Interval, ST segment, RR Interval were measured. Heart Rate (HR) can be measured from these intervals.

\subsection{Decision Making}

We have consulted with several cardiologists for the decision making process and finally came up with six decision making rules.

Sample MATLAB Code used in our program is given below:

if $\mathrm{f} 1>0 \& \& \mathrm{f} 2==0 \& \& \mathrm{f} 3==0 ; \operatorname{disp}$ ('Heart Rate Normal')

elseif $\mathrm{f} 2>0 \& \& \mathrm{f} 3==0 ; \operatorname{disp}($ 'Tachycardia')

elseif $\mathrm{f} 3>0$ \&\& $\mathrm{f} 2==0 ; \operatorname{disp}($ 'Bradycardia')

$\% \quad$ elseif $\mathrm{f} 3>0$ \&\& f2>0; disp('PACs detected')

End

if $\mathrm{f} 4>0$ \& \& f5==0 \& \& f6==0; disp('No Heart Block')

elseif f5>0; disp('Heart Block Detected')

elseif f6>0 \&\& f2==0; disp('Junctional Arrhythmia')

end

\section{RESULTS AND DISCUSSION}

The signal 103 is used from MIT-BIH database [17] to calculate different intervals. Heart rate is also calculated from different sample of that signal. These intervals for 11 samples from 103 number dataset are shown in Table 2.
Table 2: Extracted data from ECG Signal

\begin{tabular}{|l|l|l|}
\hline PR Interval & QRS Interval & $\begin{array}{l}\text { Heart Rate } \\
(\mathrm{HR})\end{array}$ \\
\hline 0.1778 & 0.0944 & 69.6774 \\
\hline 0.1778 & 0.0917 & 71.7608 \\
\hline 0.1722 & 0.0917 & 71.0526 \\
\hline 0.1722 & 0.0889 & 71.5232 \\
\hline 0.1806 & 0.0889 & 69.0096 \\
\hline 0.1861 & 0.0972 & 65.0602 \\
\hline 0.1750 & 0.0944 & 68.1388 \\
\hline 0.1694 & 0.0889 & 72.0000 \\
\hline 0.1750 & 0.0917 & 72.0000 \\
\hline 0.1722 & 0.0917 & 71.2871 \\
\hline 0.1778 & 0.0944 & 70.2580 \\
\hline
\end{tabular}

We have selected a random sample from MIT-BIH database and chosen 20 samples. Then the arrhythmias are detected using our program. They were compared with the database results and are listed below at Table 3 .

Table 3: Arrhythmia Detection from different samples

\begin{tabular}{|c|c|}
\hline S.N. & Classification \\
\hline 01 & Heart Block \\
\hline 02 & Normal \\
\hline 03 & Normal \\
\hline 04 & Junctional Arrhythmia \\
\hline 05 & Junctional Arrhythmia \\
\hline 06 & Bradycardia, Junctional Arrhythmia \\
\hline 07 & Tachycardia \\
\hline 08 & Bradycardia, Junctional Arrhythmia \\
\hline 09 & Premature Ventricular Contraction (PVC) \\
\hline 10 & Premature Ventricular Contraction (PVC) \\
\hline 11 & Premature Atrial Contraction (PAC) \\
\hline 12 & Normal \\
\hline 13 & Bradycardia,Junctional Arrhythmia \\
\hline 14 & BradycardiaJunctional Arrhythmia \\
\hline 15 & Junctional Arrhythmia \\
\hline 16 & Bradycardia, Heart Block \\
\hline 17 & Bradycardia, Junctional Arrhythmia \\
\hline 18 & Bradycardia \\
\hline 19 & Normal \\
\hline 20 & Heart Block \\
\hline
\end{tabular}


The results we've got during this proposed detection procedure was checked with the actual results. While comparing the results we've got $90 \%$ accuracy which can be increased applying further filters and using other techniques.

\section{CONCLUSION}

In this paper, we have shown a program to identify features from an ECG signal \& detect different sorts of Arrhythmia. To test our program, we have used data files (in .mat format) taken from PhysioBank ATM of MIT-BIH database. Decision making rules were made taking help from several cardiologists. Five-step procedure was taken to find the arrhythmia. The results shown here gives an easy way to detect different arrhythmias. To improve the accuracy, we need to increase the number of parameters in the decision making process. That will help us to improve the accuracy maintaining the simplicity. The future scope of this idea is to implement it practically.

\section{REFERENCES}

[1] Wen Wei and Jerry M. Mendel, "A Fuzzy Logic Method for Modulation Classification in Nonideal Environments", IEEE Transactions on Fuzzy Systems, Vol. 7, No. 3, June 1999, pp. 333-344.

[2] Tomoharu Nakashima, Gerald Schaefer, Yasuyuki Yokota, HisaoIshibuchi, "A weighted fuzzy classifier and its application to image processing tasks", Fuzzy Sets and Systems 158, 2007, pp. $284-294$.

[3] Reza Boostani, MojtabaRismanchib, Abbas Khosravani, LidaRashidi, SamanehKouchaki, PayamPeymani, SeyedTaghiHeydari, B. Sabayan, K. B. Lankarani, "Presenting a hybrid method in order to predict the 2009 pandemic influenza A (H1N1)", Advanced Computing: An International Journal (ACIJ), Vol.3, No.1, January 2012, pp. 31-43.

[4] Ken Nozaki, Hisao Ishibuchi and Hideo Tanaka, "Adaptive Fuzzy Rule-Based Classification Systems", IEEE Transactions on Fuzzy Systems, Vol. 4, No. 3, 1996, pp. 238-250.

[5] Jia Zeng and Zhi-Qiang Liu, "Type-2 Fuzzy Sets for Pattern Recognition: The State-of-the-Art", Journal of Uncertain Systems, Vol.1, No.3, 2007, pp. 163-177.

[6] F. Hoffmann, B. Baesens, J. Martens, F. Put and J. Vanthienen, "Comparing a genetic fuzzy and a Neuro- fuzzy classifier for credit scoring", presented at Int. J. Intell. Syst., 2002, pp. 1067-1083.

[7] F.M. Schleif, T. Villmann, B. Hammer, "Prototype based Fuzzy Classification in Clinical Proteomics", International Journal of Approximate Reasoning, 2008, 47(1), pp. 4-16.

[8] Aaron K. Shackelfordand Curt H. Davis, "A Hierarchical Fuzzy Classification Approach for High-Resolution Multispectral Data Over Urban Areas", IEEE Transactions on Geo-science And Remote Sensing, Vol. 41, No. 9, September 2003, pp. 1920-1932.

[9] Benoit Mandelbrot, "How long is the coast of Britain?" Science, New Series, Vol. 156, No. 3775, May 5, 1967, pp. 636-638.

[10] B. Mandelbrot, "The fractal geometry of nature", Wh. Freeman, 1983.

[11] S. Raghav and K. Misra, "Fractal Feature Based ECG Arrhythmia Classification”, IEEE, 2008, pp. 1-5.

[12] L. Y. Shyu, Y. H. Wu, and W. C. Hu, "Using wavelet transform and fuzzy neural network for VPC detection from the holter ECG," IEEE Trans. Biomed. Eng., vol. 51, no. 7, pp. 1269-1273, Jul. 2004

[13] Z. Benyd L. Czinege, Computer Analysis of Dynamic Systems with Application in Physiology, Proc IS" World congress of MACS on Scientific Computation, Modeling and Applied Mathematics, Berlin, 1997. Vol. El. pp. 663668 .

[14] V. Kecman, Learning and Soft Computing Support vector machines Neural Networks-Fuzzy Logic, MIT, 2001

[15] HA Guvenir, B Acar, Feature Selection using a Genetic Algorithm for the detection of abnormal ECG recordings, International Conference on Machine Learning, Florida, 2001.

[16] ECG Notes Interpretation \& Management Guide, Shirley A. Jones

[17] Online resource for physiological Signals. http://www.physionet.org 